\title{
Inmunoadsorción en la unidad de diálisis, ¿qué ha supuesto para enfermería?
}

\author{
Carina Caamaño Lado, Pilar Sobrado Sobrado, Azucena Barranco Sos, Ángeles Mayordomo Sanz, Alba Luz \\ Montoya Echeverry, Antonia Vives Bonjoch
}

\section{Hospital Clinic. Barcelona}

\section{Introducción:}

La inmunoadsorción es una técnica utilizada para eliminar las inmunoglobulinas (IgG, IgA, IgM e IgE) e inmunocomplejos a través de las columnas Therasorb-Ig Flex, que contienen 45 cc de sefarosa CL-4B húmeda, unida a anticuerpos de oveja anti-inmunoglobulinas humanas. Con este trabajo describiremos lo que supuso la implantación de esta nueva técnica en nuestra unidad, a nivel de dotación de personal de enfermería, de recursos materiales y aumento de carga de trabajo en la unidad.

\section{Objetivos:}

1. Describir el proceso de implantación de esta técnica en nuestra Unidad.

2. Determinar las necesidades organizativas del personal para llevar a término dicho tratamiento.

\section{Material y métodos:}

\section{Material:}

Tarjeta del paciente y de las columnas, dos columnas, set de líneas, disco separador, filtros de partículas, tapones estériles, bolsas de citrato, bolsas de PBS, bolsas de glycina, bolsas de suero fisiológico, botella PBS ázide, bombas de infusión, heparina sódica al 1\%, gluconato cálcico, material de conexión a CVC o a FAVI.

\section{Método:}

- Conocer la técnica in situ en otro hospital asistiendo la supervisora y una enfermera.
- Asistir al curso de aprendizaje, cuya duración es de 4 días.

- Día 1: Presentación de la terapia. Principio del tratamiento de Inmunoadsorción, Descripción del monitor Life $18 \mathrm{tm}$ y de las columnas TheraSorbtm Ig Flex.

- Día 2: Montaje del monitor con la enfermera instructora, explicación y resolución de posibles alarmas.

- Día 3: Preparación del monitor y realización del primer tratamiento Ig Flex con el paciente, realizado por la enfermera instructora.

- Día 4: Preparación del monitor Life 18tm y realización del segundo tratamiento por enfermería y la instructora. Revisión y evaluación de los conocimientos adquiridos a través de un cuestionario.

- Organización y adaptación del nuevo tratamiento en nuestra unidad: se organizó de forma que el tratamiento se realizara generalmente en el turno de tarde, y domingo por la mañana, en uno de los doce puestos de diálisis que consta la unidad, dado que el personal de enfermería está altamente cualificado y con experiencia en circuitos extracorpóreos.

- Para garantizar la realización de la inmunoadsorción se instruyó a seis enfermeras de todos los turnos para dar respuesta a la demanda de tratamiento. La implantación de esta técnica supuso un aumento de cargas de trabajo para el personal de enfermería. A lo largo de los tratamientos realizados han surgido dificultades, tanto a nivel técnico como en la regu- 
larización de medicación administrada, (gluconato y citrato cálcico) que se han ido superando con éxito gracias a las habilidades del equipo.

\section{Conclusiones:}

Se comprobó que al llevar a término la inmunoadsorción si bien, no es más compleja que una diálisis para el personal experto, requiere de una dedicación, minuciosidad, vigilancia y analíticas constantes. Por lo que se necesitó una enfermera para llevar en exclusividad la aféresis. Todo ello trajo consigo un aumento de implicación a nivel personal y profesional de enfermería, que se tradujo en una enorme satisfacción del trabajo realizado, siendo un estímulo para continuar en la formación de nuevas técnicas y retos. 